\title{
Internet of Things Enabled In-Home Health Monitoring System Using Early Warning Score
}

\author{
Arman Anzanpour ${ }^{1}$, Amir-Mohammad Rahmani ${ }^{1,2}$, Pasi Liljeberg ${ }^{1}$, Hannu Tenhunen ${ }^{1,2}$ \\ ${ }^{1}$ Department of Information Technology, University of Turku, Finland \\ ${ }^{2}$ Department of Industrial and Medical Electronics, KTH Royal Institute of Technology, Sweden \\ \{armanz, amirah, pakrli\}@utu.fi, hannu@kth.se
}

\begin{abstract}
Early warning score (EWS) is an approach to detect the deterioration of a patient. It is based on a fact that there are several changes in the physiological parameters prior a clinical deterioration of a patient. Currently, EWS procedure is mostly used for in-hospital clinical cases and is performed in a manual paper-based fashion. In this paper, we propose an automated EWS health monitoring system to intelligently monitor vital signs and prevent health deterioration for in-home patients using Internet-of-Things (IoT) technologies. IoT enables our solution to provide a real-time $24 / 7$ service for health professionals to remotely monitor inhome patients via Internet and receive notifications in case of emergency. We also demonstrate a proof-of-concept EWS system where continuous reading, transferring, recording, and processing of vital signs have been implemented.
\end{abstract}

\section{Keywords}

Early Warning Score, Internet of Things, Wearable electronics, Wireless Sensor Network, Body Area Network, Remote Patient Monitoring

\section{INTRODUCTION}

While the network of connected devices are growing and expanding, Internet of Things (IoT) is stepping into every aspects of life. IoT provides a competent and structured approach to improve health and wellbeing of mankind. The consequences of IoT technologies entering to medical and healthcare sectors are forming a new structured communication route between caregivers and patients [1]. In such systems, a set of connected wearable or implantable sensors continuously read patient's vital signs enabling caregivers to access the data through the Internet [2].

IoT-enabled health monitoring systems often work in a way that wireless body area network (WBAN), which is a set of medical sensors attached to patient's body, records physiological parameters and vital signs[3] and sends them to a cloud server for further processing and storage. Considering each sensor in WBAN as an IP-based connected node, an IoT-enabled healthcare system offers the opportunity to serve patients needing continuous monitoring outside hospital environment.
It has been reported that the number of critically ill patients is growing and many patients leaving hospital are still at the risk of deterioration at home [4]. Several of those patients might encounter health deterioration when there are some abnormal changes appearing in their vital signs [5]. In order to predict health deterioration, a technique called Early warning score (EWS) [6] has been proposed. In this system, nurses record the patients' vital signs in an observation chart at certain time intervals and assign a score to the value of each sign in its range. The overall patient score, which is the sum of all individual scores, is then used to decide whether the patient is deteriorating or not[7]. The process of recording and calculating EWS is usually still paperbased and manual in hospitals. Paper-based and inaccurate data collection leads quite often to incorrect calculation of warning score making health experts to misdiagnose the situation. Furthermore, manual paper-based approach is slow and requires work-time resources form the caregivers. Recently, early warning systems have begun to move toward automated electronic platforms [8]. IoT-enabled wearable sensor networks can help the process of EWS automation to be extended for in-home use cases.

In this paper, we propose and demonstrate an EWS health monitoring system to automatically predict and prevent health deterioration for in-home patients. To the best of our knowledge, ours is the first proposal for bringing and utilizing EWS for automated in-home scenarios. The ultimate objective of this work is to provide a full IoT-based EWS monitoring system including all the system components from WBAN as an all-in-one convenient device to use cloud services. With this implementation, we will be able to extend the utilization of early warning score to homes, and hence offering a more efficient way of remote patient monitoring.

The rest of this paper is structured as follows. Section 2 describes the early warning score methods. In Section 3 , the related work is reviewed. Section 4 provides the system architecture and Section 5 is for demonstration and evaluation. Finally, Section 6 concludes the document.

\section{EARLY WARNING SCORE}

Early Warning System is a methodology for early detection of health deterioration to minimize the impact of sudden severe changes in health. Such a system uses a process called Early Warning Score (EWS) to calculate different scores from patient's observation chart based on repetitive physiological measurement of vital signs to derive a composite score which is used to identify if a patient is at a risk of deterioration. Studies in this field have shown that patients often have signs of clinical deterioration up to 24 hours prior to a serious clinical case requiring a full intervention [9][10][11]. The simplest type of score can be calculated 
Table 1: A typical early warning score model [4]

\begin{tabular}{|l|c|c|c|c|c|c|c|}
\hline Physiological parameters & $\mathbf{3}$ & $\mathbf{2}$ & $\mathbf{1}$ & $\mathbf{0}$ & $\mathbf{1}$ & $\mathbf{2}$ & $\mathbf{3}$ \\
\hline Respiration rate (breaths per minute) & $\leq 8$ & & $9-10$ & $12-20$ & & $21-24$ & $\geq 25$ \\
\hline Oxygen saturation (\%) & $\leq 91$ & $92-93$ & $94-95$ & $\geq 96$ & & & \\
\hline Temperature (oC) & $\leq 35.0$ & & $35.1-36.0$ & $36.1-38.0$ & $38.1-39.0$ & $\geq 39.1$ & \\
\hline Systolic BP (mmHg) & $\leq 90$ & $91-100$ & $101-110$ & $111-219$ & & & $\geq 220$ \\
\hline Heart rate (beats per minute) & $\leq 40$ & & $41-50$ & $51-90$ & $91-110$ & $111-130$ & $\geq 131$ \\
\hline Level of consciousness & & & & $\mathrm{A}^{*}$ & & & $\mathrm{~V}, \mathrm{P}$ or $\mathrm{U}^{*}$ \\
\hline
\end{tabular}

* $\mathrm{A}=$ Alert, $\mathrm{V}=$ response to voice, $\mathrm{P}=$ response to pain, $\mathrm{U}=$ unresponsive

using five physiological parameters: level of consciousness, pulse rate, systolic blood pressure, respiration rate, body temperature, and blood oxygen saturation. Each parameter has a maximum score of 3 and a minimum score of 0 from which the final score can be calculated. Table 1 shows a typical early warning score model. After calculating the early warning score, the treatment order of the patient can be changed based on the score results. Lower scores lead to change in observation frequency and higher scores results is providing a higher level of medical care for the patient such as assessment by health expert or transferring to the intensive care unit.

\section{RELATED WORK}

Early warning score as a method for preventing health deterioration was proposed for the first time in 1997 [12]. This preliminary EWS method changed several times during the application in hospitals to enhance and optimize the algorithm and process [13]. The outcome was a modified early warning system (MEWS) [14], a standardized version (SEWS) [15], and finally national early warning scores (NEWS) [16]. The main difference among these methods is regarding the number of recorded medical parameters and also the score of each vital sign in the range of values. The concept of EWS is in use in several countries such as Sweden [17] and New Zealand [18][19] with some modification. Although the use of this scoring system has been extremely useful for hospitals in terms of saving lives and reducing the cost of healthcare, there have been some reports about failures due to poor quality data collection and inaccurate EWS score calculation, forcing many hospitals to use electronic vital signs recording [8].

There are some commercially available electronic EWS systems for hospitals. A system named IntelliVue Guardian Solution[20][21] is using EWS which detects the patients in a hospital at risk of deterioration, helps caregivers to avoid costly transfer or readmission to the ICU. Another hospital based vital signs recording system is the Remote Service for Diagnostics. [22] It is a remote monitoring system for the medical experts to control the devices and vital signs recorders via internet to monitor the patients in a hospital. These systems are designed to work only with hospital monitoring systems and in-home usability and wearability are not considered.

There exists also several products available in the market to be used by patients. Although most of these products are efficient in recording medical parameters, they cannot be considered as an IoT based solution as they are mostly just standalone products. For instance, V-Patch [23] is a biosensor that is attached to the skin to enable monitoring of patients and allows collection of vital signs over a wireless network. Other similar devices such as LifeMonitor [24] and BioHarness 3 [25] can be categorized in the class of standalone vital signs recorders. ViSi Mobile [26] in turn is a in-hospital wireless vital signs measurement device.
The novelty of our proposed solution in this paper is due the extension of EWS utilization to in-home scenarios by exploiting the Internet of things technologies and demonstrating a proof-of-concept system prototype.

\section{SYSTEM ARCHITECTURE}

The system architecture of our IoT-based early warning system is shown in Figure 1. The system architecture includes 3 main components:

1)Sensor Network: In the sensor network medical parameters are recorded by wearable sensors. Sensors are categorized into three groups based on their data conversion rate and their function. The first group of sensors includes high date rate sensors used for streaming-like real-time parameters (e.g., ECG signal). The second group comprised of sensors that read and record data at a lower data rate such as body temperature and environment sensors. The last group includes sensors which are not fully automated and are periodically used by patients or home caregivers. The values read by these sensors (e.g., blood pressure) are added to the system manually.

2)e-Health Gateway: The gateway, receives data from several sensors, performs protocol conversion, and provides some services such as data compression and storage in offline mode. At the gateway layer, there can be two different kinds of approaches depending on the availability of Internet connection. If the e-Health gateway has the Internet access, it will send the real-time non-pre-processed signals (e.g., ECG signal) to the cloud server. In this approach, other data from the first sensor type can be pre-processed and the numerical results along with other data from the second type sensors will be sent to the cloud server. In case unavailability of Internet at the gateway, the e-Health gateway will store the compressed raw data and results of local pre-processings in its local database. As soon as the gateway is reconnected to Internet, it will send first the calculated values and then compressed raw data packages to the cloud server.

3)Back-End System: The back-end system has two different sections: 1) a cloud-based back-end infrastructure including data storage, data analysis, decision making, etc, 2) user interface which acts as a dashboard for medical caregivers together with performing user control and data visualization. The cloud server receives different types of sensor data using corresponding protocol and stores it in the database. At this stage, early warning score is computable when the server has received every single part of parameters needed for estimation of EWS and based on the final score, proper alerts will fire in case of emergency. Cloud server is also responsible for providing the administration control panel for health experts with real-time health data visualizers and user interface for patients and in-home caregivers. The flowchart of the cloud server functions is shown in Figure 2. 


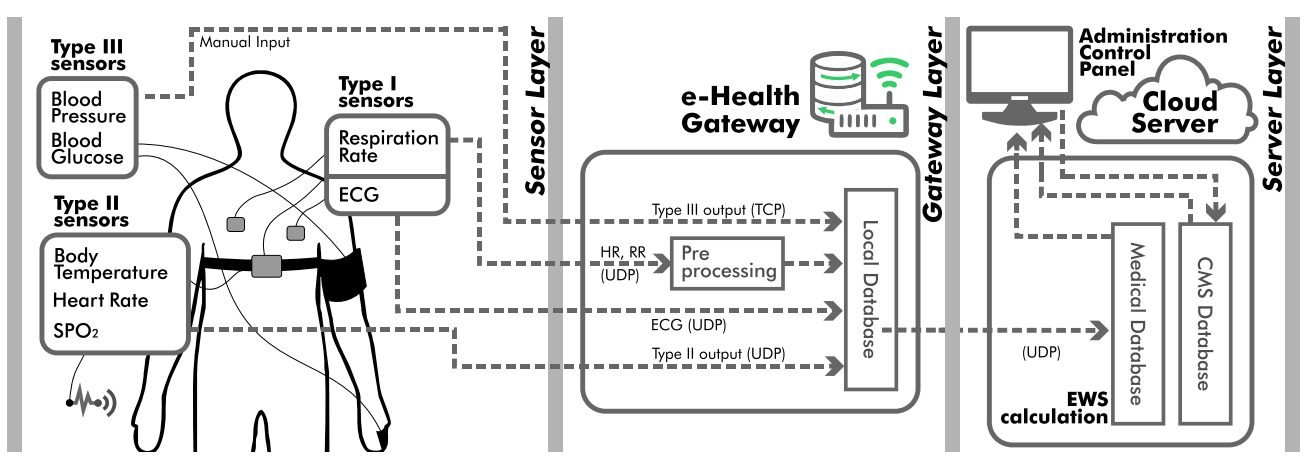

Figure 1: Architecture of the proposed IoT-based clinical early warning system

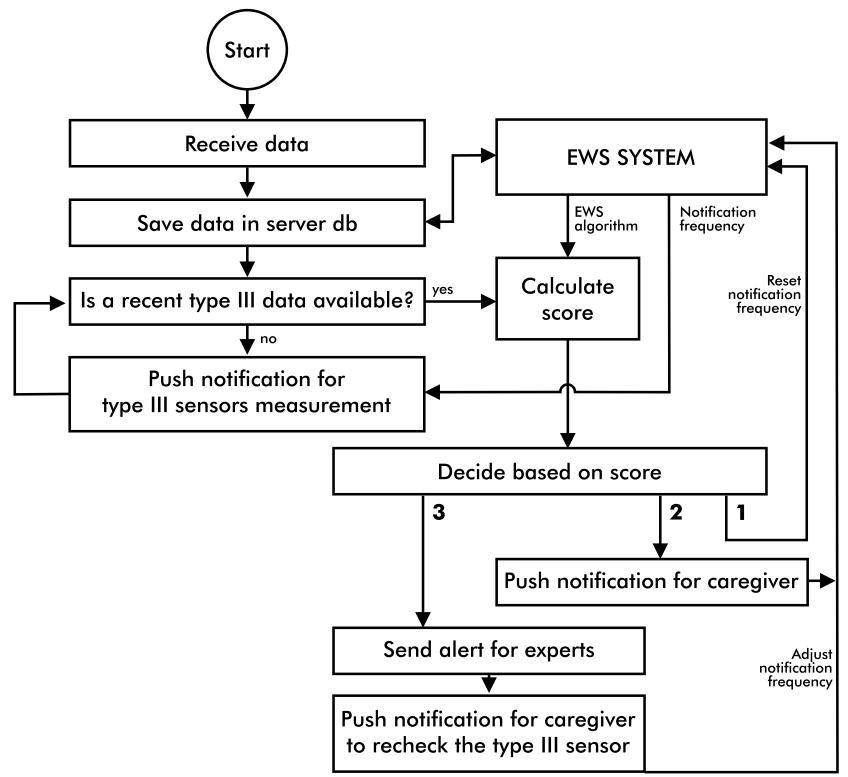

Figure 2: Cloud server and EWS system functions

\section{DEMONSTRATION AND EVALUATION}

In this section, we demonstrate our EWS system prototype. A collection of analog and digital sensors are used to set up EWS system. A pulse oximeter fingertip grip is used to record the blood oxygen saturation and heart rate simultaneously and a waterproof DS18B20 digital temperature sensor is used to record the body temperature in real-time. A thermal airflow analog sensor is used to measure the respiration rate which captures an analog signal with peaks at exhalation moments. An Arduino board is used to read the analog and digital signals from sensors and provide the serial output for a Wi-Fi module.

We use ESP8266 Wi-Fi[27] module for wireless data transmission. It is a tiny yet powerful and low-cost Wi-Fi module with several features such as GPIO pins, 10-bit ADC, SPI, I2C, PWM, and a microcontroller running at $80 \mathrm{MHz}$ with $64 \mathrm{KBy}$ tes of RAM. The original firmware of this module is designed to receive Attention (AT) commands from the serial port. However, we made a custom firmware for this module enabling it to read data packets continuously from serial input and send to the e-Health gateway using UDP protocol. As we need an analog to digital converter with a higher sampling rate and a faster $\mathrm{Wi}-\mathrm{Fi}$ module to display the ECG signal in the real-time control panel, we use Texas Instruments' ADS1192 analog front end together with
RTX4140 Wi-Fi module to read and send ECG signal. A summary of sensors description is described in Table 2.

We also use a Bluetooth-based upper arm blood pressure monitor (iHealth BP5) with the capability of starting the measurement with direct command from a smart device (e.g., phone or tablet). At this level, a patient is supposed to send the blood pressure measurement manually and periodically to the system.

We develop a mobile application to receive notifications from the cloud server and transfer the manually collected data to the e-Health gateway. This mobile application is developed using PhoneGAP which is a multi-platform development tool making the application compatible with the most mobile operating systems (e.g. Android, iOS, Windows Phone, and Tizen). This application connects to the local Wi-Fi network and receives the cloud server notifications via the e-Health gateway.

The gateway is a Jetson TK1 NVIDIA board which is a powerful Linux-operated system with $2.32 \mathrm{GHz}$ ARM quadcore Cortex-A15 CPU, 192 SM3.2 CUDA cores GPU, 2 Gigabyte RAM, and 16GB fast eMMC storage. On this board, Ubuntu Linux runs a web server using Apache, a UDP server using Node.js for receiving the data from sensors and a UDP server for sending data to the cloud server. Apache server uses MySQL for storing data in the off-line mode. Data transfer between sensors and the e-Health gateway is handled using PHP on the Apache webserver and JavaScript on Node.js server.

For preliminary implantation of the EWS system, we use a virtual private server with $4 \mathrm{~GB}$ RAM, 4 Intel Xeon $3.30 \mathrm{GHz}$ CPUs, and 100GB disk space. The software configuration of this server which is operated with CentOS Linux is similar to the e-Health gateway except that it contains the main EWS code and stores all the vital signs of patients. This server continuously calculates the early warning score using the figures and thresholds reported in Table 1 and asserts appropriate alerts according to the result. We also developed a web interface (Figure 3) to display the real-time vital signs in the browser using WebSocket for requesting and receiving data from the cloud server. The data reported in the Figure 3 was captured from a 34 years old healthy male.

\section{CONCLUSIONS AND FUTURE WORK}

In this paper, we presented a solution to expand the early warning score (EWS) system beyond the hospital beds and into patients' homes. To take advantage of the benefits of EWS, we claimed that patients do not necessarily need to be monitored in hospitals, a well-structured automated so- 
Table 2: Summery of sensors

\begin{tabular}{|l|l|l|l|l|l|}
\hline Sensor Name & Description & Type & Sampling Rate & Interface & Transmission Protocol \\
\hline Body Temperature & DS18B20 & Digital & $1 \mathrm{~s} /$ second & (1-Wire) Serial & Wifi/UDP \\
\hline Heart Rate & CMS50DL & Digital & $1 \mathrm{~s} /$ second & Serial & Wifi/UDP \\
\hline Oxygen Saturation & CMS50DL & Digital & $1 \mathrm{~s} /$ second & Serial & Wifi/UDP \\
\hline Respiration Rate & Thermal Airflow & Analog & $200 \mathrm{~s} /$ second & (A2D) Serial & Wifi/UDP \\
\hline Systolic Blood Pressure & iHealth BP5 & Manual & $1-4 \mathrm{~s} /$ hour & Bluetooth & Wifi/TCP \\
\hline ECG & ADS1192 & Analog & 1000 s/second & SPI & Wifi/UDP \\
\hline
\end{tabular}

* Based on the previous calculated early warning score

EWS Real-Time Control Panel Real Time ECG Chart
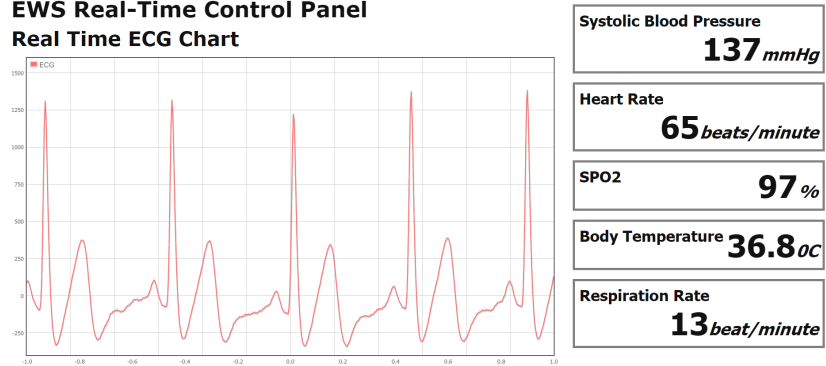

Figure 3: Live control panel web interface

lution can be utilized for patient to be monitored remotely. We demonstrated the possibility of collecting vital signs remotely on the basis of Internet of Things technologies and hence enabling in-home patients to be monitored continuously by health experts and early warned when deterioration is detected. In the future, our work will be extended by offering a more comprehensive and automatic solution considering also environmental parameters. The body area sensor network will be more convenient, wearable, and even washable. We intend to validate the system in hospital trials and make the EWS algorithm more intelligent and patient specific, so that the results will be based on the patient's medical history as well.

\section{REFERENCES}

[1] RSH Istepanian et al. Internet of m-health Things "m-IoT". In IET Sem. on Assisted Living, pages 1-3, 2011.

[2] F Fernandez et al. Opportunities and challenges of the internet of things for healthcare: Systems engineering perspective. In MobiHealth 2014, pages 263-266, Nov 2014.

[3] OC Omeni et al. Energy efficient medium access protocol for wireless medical body area sensor networks. In Medical Devices and Biosensors, Intl. Summer School and Sym. on, pages 29-32, Aug 2007.

[4] D Georgaka et al. Early warning systems. Hospital Chronicles, 7(1 Sup):37-43, 2012.

[5] D Atkinson. Nursing observation and assessment of patients in the acute medical unit. http://usir.salford.ac.uk/29466/, 2013.

[6] Una Kyriacos et al. Monitoring vital signs using early warning scoring systems: a review of the literature. $J$ Nurs Manage, 19(3):311-330, 2011.

[7] T O'Kane et al. Mews to e-mews: From a paper-based to an electronic clinical decision support system. In European Conf. on Information Management, page 301, January 2010.

[8] A Zarabzadeh et al. Features of electronic early warning systems which impact clinical decision making. In CBMS 2012 Intl. Sym., pages 1-4, 2012.
[9] J McGaughey et al. Outreach and early warning systems (ews) for the prevention of intensive care admission and death of critically ill adult patients on general hospital wards. Cochrane Database Syst Rev, (3), 2007.

[10] C Franklin et al. Developing strategies to prevent inhospital cardiac arrest: analyzing responses of physicians and nurses in the hours before the event. Critical care medicine., 22(2):244-247, February 1994.

[11] RM Schein et al. Clinical antecedents to in-hospital cardiopulmonary arrest. Chest., 98(6):1388-1392, December 1990.

[12] Morgan et al. An early warning scoring system for detecting developing critical illness. Clin Intensive Care, 8(2):100, 1997.

[13] U Kyriacos et al. Monitoring vital signs: Development of a modified early warning scoring (mews) system for general wards in a developing country. PLOS ONE, 9(1), 2014.

[14] R Paterson et al. Prediction of in-hospital mortality and length of stay using an early warning scoring system: clinical audit. Clin Med., 6(3):281-284, 2006.

[15] GD Barlow et al. Standardised early warning scoring system. Clin Med., 6(4):422-423, 2006.

[16] Royal College of Physicians. National early warning score (news): standardising the assessment of acute-illness severity in the nhs., 2012.

[17] Management Team, Sundsvall-HÃd'rnÃüsand Hospital, Sweden. Modified early warning score mews, 2011-02-07.

[18] Wellington ICU. Early Warning Scores \& Medical Emergency Teams, (accessed 2015-06-26). http://www . wellingtonicu.com/AboutUs/Services/EWS/.

[19] A Psirides et al. A review of rapid response team activation parameters in new zealand hospitals. Resuscitation, 84(8):1040 - 1044, 2013.

[20] Koninklijke Philips N.V. (accessed 2015-06-26). http://www.usa.philips.com/healthcare-articles/ early-warning-scoring-tools-for-rapid-response.

[21] Rinaldo Bellomo, Austin Hospital, Melbourne, Australia. Well-implemented early warning scores can help rapid response teams in improving outcomes, 2012.

[22] Siemens Healthcare Diagnostics Inc. (accessed 2015-06-26). http://usa.healthcare.siemens.com/ diagnostics-it/remote-monitoring/realtime-solutions.

[23] Intelesens Ltd. (accessed 2015-06-26). http://www.intelesens. com/inhomemonitoring/vpatch.html.

[24] Equivital Inc. (accessed 2015-06-26). http: //www.equivital.co.uk/products/tnr/sense-and-transmit.

[25] Zephyr Technology Corp. (accessed 2015-06-26). http://zephyranywhere. com/products/bioharness-3/.

[26] Sotera Wireless, Inc. (accessed 2015-06-26). http://www.visimobile.com/overview/.

[27] Espressif Systems. (accessed 2015-07-10). http://espressif.com/en/products/esp8266/. 